\title{
DESENVOLVENDO COMPETÊNCIAS POR MEIO DA INTERAÇÃO COM ATORES EXTERNOS EM ECOSSISTEMAS DE INOVAÇÃo AmBIENTALMENTE SuSTENTÁveIS
}

Arthur Marcon (arthur.marcon@ufrgs.br) - Programa de Pós-Graduação em Engenharia de Produção, Universidade Federal do Rio Grande do Sul (UFRGS).

José Luis Duarte Ribeiro (ribeiro@ producao.ufrgs.br) - Programa de Pós-Graduação em Engenharia de Produção, Universidade Federal do Rio Grande do Sul (UFRGS).

\section{RESUMO}

Este artigo adota uma abordagem de ecossistemas de inovação com o objetivo de analisar como as empresas que criam inovações ambientalmente sustentáveis desenvolvem competências por meio das suas interações com atores externos. Assim, usando uma abordagem qualitativa, quatro casos de empresas que desenvolveram inovações sustentáveis foram estudados para analisar como o ecossistema de inovação ambientalmente sustentável afeta o desenvolvimento de competências da empresa. Os dados foram coletados por meio de entrevistas em profundidade e analisados através de análise de conteúdo. Os achados dão conta de que os ecossistemas de inovação ambientalmente sustentáveis podem adicionar competências técnicas, de inovação, de mercado, de sustentabilidade e de colaboração ao processo de inovação das empresas. Nossos resultados contribuem para a literatura sobre inovação sustentável, fornecendo noções sólidas do impacto do ecossistema de inovação nas empresas.

Palavras-chave: inovação verde; stakeholders; universidade; incubadoras; sustentabilidade 


\section{INTRODUÇÃO E BACKGROUND TEÓRICO}

O desenvolvimento de inovações tornou-se um grande alvo para as indústrias que visam capturar retornos financeiros e se tornarem líderes em tecnologia (ADNER; KAPOOR, 2010). No entanto, inovar frequentemente requer conhecimento e capacidades que as empresas não detêm por completo e, portanto, estratégias de inovação aberta que incluem atores e entidades externas podem ser de grande ajuda (LEE, 2007; MARCON; RIBEIRO, 2021; MEDEIROS et al., 2018;). Inovação é um processo sistêmico e, como tal, a continuidade no mercado e o sucesso da inovação precisam vir acompanhadas de mudanças no ambiente que deem suporte a sua existência e aumentem a funcionalidade e, consequentemente, o valor entregue (ADNER; KAPOOR, 2010). Para tanto, as empresas estabelecem redes de parceiros para aumentar a amplitude e a profundidade de suas invenções em ecossistemas de inovação (MARCON; RIBEIRO, 2021; NAMBISAN; BARON, 2013;).

Conceitualmente, várias definições de ecossistema foram propostas (ver GOMES et al., 2018). Muitos teóricos afirmam que o nascimento do conceito de ecossistema de inovação pertence a Moore (1993), que propôs que uma empresa pode ser vista como parte de um ecossistema de negócios que não está restrito às suas fronteiras geográficas ou setoriais e que é caracterizado pela interdependência e coevolução de capacidades para aumentar o valor da inovação. Granstrand e Holgersson (2020) definem ecossistemas de inovação como o conjunto de atores, atividades, artefatos, e instituições e relações que evoluem e são importantes para o desempenho inovativo de um ator ou de uma população de atores. Gomes et al. (2018) destaca a evolução do conceito que partiu de um foco na captura de valor (ecossistema de negócios, como proposto por MOORE, 1993) para a cocriação de valor (ecossistemas de inovação, como proposto por ADNER, 2006).

Considerando a importância dos ecossistemas de inovação para o desenvolvimento de novos produtos, serviços e processos, os ecossistemas de inovação ambientalmente sustentáveis (EIAS) surgem como um importante tema de interesse, uma vez que inovações sustentáveis exigem um esforço conjunto de fornecedores, universidades, governantes, consumidores e demais atores para ter sucesso e promover a mudança para economias mais sustentáveis (FAGERBERG, 2018, MARCON et al., 2021).

Portanto, gerenciar vários interesses e diferentes capacidades, e ao mesmo tempo reconhecer oportunidades de inovação e crescimento são desafios críticos para o sucesso dos ecossistemas (NAMBISAN; BARON, 2013; TSUJIMOTO et al., 2017; ZENG; HU; 
OUYANG, 2017). Somado a isso, EIAS também deve incluir interesses de sustentabilidade no desenvolvimento e comercialização de inovações ( ZENG; HU; OUYANG, 2017). As questões ambientalmente sustentáveis são reconhecidas por irem além das competências centrais (core competences) da maioria das empresas (MOUSAVI; BOSSINK, 2017). Tal fator ressalta ainda mais a necessidade da implantação de práticas de inovação aberta que conhecimentos externos possam fluir para dentro das empresas de modo a permitir que elas sejam capazes de desenvolver inovações sustentáveis (MARCON et al., 2021). Alinhado a isso, tecnologias complementares e concorrentes, políticas de inovação, interação entre atores e capacidades organizacionais constituem importantes fatores de influência nos ecossistemas de inovação (MARCON; RIBEIRO, 2021).

No entanto, as pesquisas até agora não abordaram como os EIAS são organizados e como suas partes se relacionam para possibilitarem interações bem-sucedidas (FAGERBERG, 2018; YAP; TRUFFER, 2018). Em um dos poucos artigos de pesquisa acadêmica que abordou EIAS, Zeng, Hu e Ouyang (2017) estudaram a relação paradoxal entre exploração e aproveitamento do conhecimento em um EIAS. Enquanto Marcon et al., (2021) analisaram os atores que compõem EIAS. Dessa forma, os mecanismos que sustentam os EIAS ainda são desconhecidos e as pesquisas não avançaram no sentido de elucidar como os EIAS se organizam e como eles são afetados pelas políticas, organizações e instituições de inovação (YAP; TRUFFER, 2018). Além disso, embora a literatura sobre inovação sustentável reconheça a importância da inovação aberta para o desenvolvimento de inovações sustentável, os pesquisadores não empregaram a lente dos ecossistemas de inovação para elucidar as faces desse tema.

Assim, com base no potencial de contribuição da visão de ecossistema de inovação para a compreensão das dinâmicas de inovação sustentável, este artigo aborda ecossistemas de inovação ambientalmente sustentáveis. Assim, pretende-se compreender como as empresas desenvolvem competências através dos EIAS desenvolvidos em torno das inovações sustentáveis. Desta forma, analisou-se empresas que desenvolveram inovações sustentáveis utilizando práticas de inovação aberta para entender os EIAS e como eles influenciam no desenvolvimento de competências por parte das empresas-focais. Os objetivos propostos estão alinhados com a preocupação por uma economia mais ambientalmente sustentável baseada em inovações e consumo sustentável (MARCON; MEDEIROS; RIBEIRO, 2017). 


\section{MÉTODO}

Como o objetivo deste artigo é compreender como os EIAS se estruturam, ainda um tema com limitados insights empíricos, optou-se por adotar uma abordagem qualitativa do problema, em consonância com o estudo de Ben Arfi, Hikkerova e Sahut (2018) e as proposições de Rosenthal (2016). Assim, foram conduzidos estudos de caso em profundidade de quatro empresas que desenvolvem inovações ambientalmente sustentáveis e que interagem ativamente com seus EIAS. Os dados foram coletados principalmente por meio de entrevistas semiestruturadas para obter percepções mais aprofundadas sobre a dinâmica do EIAS de cada empresa, e foram complementados por fontes de dados das empresas para enriquecer os resultados, tais como perfis em redes sociais de negócios, reportagens, releases de imprensa e vídeos (EISENHARDT; GRAEBNER, 1989).

Para garantir representatividade e generalização dos achados, foram selecionadas empresas de diferentes portes, estruturas, setores e níveis de maturidade da inovação. A decisão por uma análise qualitativa de estudos de caso deriva das recomendações de Voss et al. (2002), que sugere a utilização de estudos de caso quando o objetivo dos pesquisadores é explorar um novo fenômeno e construir teoria a partir dele. Nesse sentido, este trabalho utiliza as empresasfocais como unidades de análise para ajudar a compreender o EIAS que as rodeia, uma vez que as empresas-focais são os atores que detém a maior parte do conhecimento e das informações sobre o ecossistema de inovação.

Para garantir que a empresa selecionada atendesse aos requisitos desta pesquisa e pudesse contribuir com o tema em estudo, foi feito um contato preliminar, onde possíveis respondentes foram questionados sobre inovações ambientalmente sustentáveis, a participação de atores do EIAS no processo de inovação entre outros aspectos. Assim, foram selecionados quatro casos de empresas que desenvolveram inovações ambientalmente sustentáveis e, para cada caso, a pessoa mais adequada para responder ao questionário foi entrevistada. $\mathrm{O}$ questionário continha nove perguntas que abordavam inovações ambientalmente sustentáveis, estrutura e governança do ecossistema, e o impacto do EIAS na empresa. Para garantir o anonimato, atribuímos codinomes aos casos estudados (Trator, Simulação em saúde, Varejo de moda, e Secagem de grãos). Uma breve descrição de cada empresa é apresentada na Tabela 1, que resume as informações sobre cada caso. 
Tabela 1 - Informações sobre os casos estudados

\begin{tabular}{|l|l|l|l|}
\hline Codinome & Porte & Entrevistado & Descrição da inovação \\
\hline Trator & $\begin{array}{l}\text { Grande } \\
\text { empresa }\end{array}$ & $\begin{array}{l}\text { Gerente de Engenharia } \\
\text { de Produto }\end{array}$ & Trator com emissão residual controlada \\
\hline $\begin{array}{l}\text { Simulação } \\
\text { em Saúde }\end{array}$ & Startup & CEO/Fundadora & $\begin{array}{l}\text { Dispositivos e produtos para simulação em saúde, como } \\
\text { frascos, géis e manequins }\end{array}$ \\
\hline $\begin{array}{l}\text { Varejo de } \\
\text { moda }\end{array}$ & $\begin{array}{l}\text { Grande } \\
\text { empresa }\end{array}$ & $\begin{array}{l}\text { Analista de } \\
\text { Sustentabilidade }\end{array}$ & $\begin{array}{l}\text { Desenvolvimento de uma cadeia sustentável de varejo } \\
\text { de moda para promover a sustentabilidade ambiental por } \\
\text { meio de matérias-primas e processos ambientalmente } \\
\text { sustentáveis }\end{array}$ \\
\hline $\begin{array}{l}\text { Secagem de } \\
\text { grãos }\end{array}$ & Startup & CEO/Fundador & $\begin{array}{l}\text { Desenvolvimento de soluções sustentáveis para secagem } \\
\text { de grãos, eliminando a necessidade de fontes de calor } \\
\text { altamente poluentes durante a secagem dos grãos }\end{array}$ \\
\hline
\end{tabular}

Fonte: Os autores (2021)

As entrevistas foram gravadas e transcritas. Utilizou-se a técnica de análise de conteúdo por meio de codificação para destacar o conteúdo relevante das transcrições em categorias, assim como feito anteriormente no artigo de Ben Arfi et al (2018). Os trechos que abordam os temas desta pesquisa receberam códigos que permitiram rastrear conteúdos relevantes nas entrevistas.

\section{RESULTADOS}

O desenvolvimento de inovações ambientalmente sustentáveis requer competências que nem sempre as empresas detêm. Assim, nesta seção são reportadas as competências desenvolvidas pelas empresas ou integradas ao processo de inovação derivadas das interações com outros atores do EIAS.

\subsection{Competências Técnicas}

O desenvolvimento de inovações ambientalmente sustentáveis geralmente requer a mudança do design do produto ou das funcionalidades e dos processos industriais. Nesse sentido, os casos analisados relataram que o EIAS ajudou as empresas a desenvolverem novas competências técnicas para projetar (design) e fabricar produtos ambientalmente sustentáveis. Essas competências técnicas derivam principalmente de universidades, fornecedores, consultorias e especialistas. Por exemplo, o caso Secagem de grãos buscou conhecimento técnico sobre agronomia e armazenamento de grãos por meio da colaboração com uma 
universidade, uma empresa de tecnologia e especialistas na área. A falta de um produto pronto para o mercado e a necessidade de validar a proposta do modelo de negócios levaram a empresa a estabelecer parcerias para a aquisição de tal conhecimento.

O caso da empresa Simulação em saúde destaca competências agregadas por especialistas. Diferentemente do caso de Secagem de Grãos, Simulação em saúde buscou o conhecimento de especialistas para desenvolver os protótipos iniciais dos produtos de simulação, uma vez que o startup não tinha conhecimento das características técnicas e processos necessários para desenvolver substâncias químicas.

Na época, eu conversei com químicos, biólogos, enfermeiras, farmacêuticos porque eu não tinha conhecimento técnico sobre desenvolvimento de géis. Meu background acadêmico é em enfermagem, então eu não tinha treinamento nisso, porém eu sabia aonde queria chegar, e então fui atrás de quem poderia me ajudar.

(CEO/Fundadora, Simulação em saúde)

\subsection{Competências para Inovação}

Nossos resultados mostram que os EIAS também desenvolvem ou melhoram as competências para inovação das empresas, ou seja, ao desenvolver inovações ambientalmente sustentáveis, as empresas desenvolvem maior maturidade em inovação. Dessa forma, elas são capazes de explorar possibilidades de inovação derivadas do conhecimento adquirido com a inovação ambientalmente sustentável desenvolvida e expandir tal capacidade para outros produtos ou aplicações. Nesse sentido, por exemplo, a empresa Varejo de moda utilizou o conhecimento adquirido na formação da sua cadeia de suprimentos sustentável para produtos jeans para aplicar e desenvolver uma cadeia de suprimentos sustentável para os produtos de malha. Isso é evidenciado no seguinte trecho:

Após transformarmos a cadeia do jeans em sustentável, decidimos fazer isso também para a cadeia da malha. Assim, a empresa começou a coletar resíduos dos fabricantes, levá-lo para fazer a desfibragem e então conduzir todo o processo exatamente como na cadeia do jeans.

(Analista de Sustentabilidade, Vareja de moda)

Além disso, essas empresas passam a explorar inovações e melhorias de produto a partir da inovação ambientalmente sustentável para desenvolver novas inovações. O caso Trator é um exemplo disso. Quando a empresa desenvolveu o novo motor, tais inovações permitiram 
adicionar novas peças ao trator como um todo e melhorar sua eficiência e possibilidade de aplicações. O gerente de engenharia de produto da empresa explica isso no trecho a seguir:

Em resposta às interações que ocorrem nesses ecossistemas, as coisas começaram a acontecer. Conforme você começa a abrir novos caminhos, novas possibilidades surgem cada vez mais. Hoje existem máquinas agrícolas com freio ABS, suspensão ativa etc. que permitem ganhos. Dependendo da temperatura do motor, a hélice de resfriamento do radiador pode ser ligada ou desligada.

(Gerente de Engenharia de Produto, Trator)

\subsection{Competências de mercado}

Além das competências para inovação, o EIAS ajuda as empresas a desenvolver competências de mercado. Competências de mercado estão relacionadas a um melhor entendimento acerca dos consumidores e de suas demandas. Essas competências podem ser desenvolvidas integrando o consumidor durante o desenvolvimento do produto ambientalmente sustentável, o que foi feito pela empresa Secagem de grãos, ou por meio de parcerias com fornecedores como nos casos Varejo de moda e Simulação em saúde. O trecho a seguir da CEO e fundadora da empresa Simulação em saúde relata as competências de mercado desenvolvidas durante o desenvolvimento da inovação e sua prototipagem e teste:

Eu preciso do meu cliente, a opinião dele é muito importante; nesse momento estamos prototipando, então estamos testando o gel com consumidores. As vezes o revendedor também participa. Um revendedor me sinalizou essa necessidade e pediu "Faça o gel pois há demanda de mercado." Eu fiz o gel para ele testar, e ele me disse que aquele gel havia falhado pois não era apropriado para ser usado em manequins (...). Então, eu tive que adaptar a fórmula e testar múltiplas vezes com ele”.

(CEO/Fundadora, Simulação em saúde)

\subsection{Competências de sustentabilidade}

EIAS são especialmente importantes para o desenvolvimento de competências de sustentabilidade. Como o desenvolvimento de inovações sustentáveis requer conhecimentos relacionados a materiais e suas propriedades, avaliação de sustentabilidade e modificações de processos industriais, os atores do ecossistema fornecem esse conhecimento para empresasfocais para permitir o desenvolvimento de inovações tanto em processos quanto em produtos finais. O trecho apresentado a seguir fornece alguns exemplos dessa dinâmica: 
Também fizemos uma interface com a academia e empresas sustentáveis. Temos parcerias com diversas universidades. A academia nos trouxe conhecimento de análise do ciclo de vida, por exemplo, que não tínhamos internamente.

(Analista de Sustentabilidade, Vareja de moda)

Isso acontece mesmo quando a empresa detém algum conhecimento interno sobre sustentabilidade. Um exemplo dessa dinâmica de inovação aberta "de fora para dentro" (inbound) pode ser observado no caso da empresa de varejo de moda. A empresa possui um departamento interno para aspectos de sustentabilidade e incluiu universidades e fornecedores no desenvolvimento de produtos sustentáveis e de uma cadeia de suprimentos mais sustentável para adquirir conhecimento externo. Portanto, os atores externos atuaram como provedores de conhecimento, enquanto o conhecimento interno detido pela empresa e seus funcionários foi responsável por fazer a tradução do conhecimento externo de modo a permitir que a empresa aplique e explore as (novas ou aprimoradas) competências em inovações ambientalmente sustentáveis.

\subsection{Competências de colaboração}

Por fim, os resultados também demonstram que o EIAS fornece às empresas competências de colaboração. As competências de colaboração ajudam as empresas a tirar melhor proveito da sua participação no EIAS, uma vez que são mais capazes de adquirir novos conhecimentos e construir relacionamentos mais próximos com os parceiros (especialmente fornecedores). Essa competência foi observada no caso do Varejo de moda, que é uma grande varejista de roupas e produtos de moda e, devido ao seu grande porte, toda a cadeia de suprimentos seguia seus "comandos", o que muitas vezes implicava em comportamentos oportunistas da própria empresa. Com a maior pressão pela sustentabilidade e a adoção dos valores da empresa voltados para a sustentabilidade, o EIAS obrigou a empresa a colaborar mais com os parceiros para garantir um engajamento de sucesso. Isso fez com que a empresa ficasse mais atenta às necessidades e realidades dos parceiros e, assim, possibilitou a criação de empatia para com os parceiros. Ou seja, a empresa passou a ser capaz de desenvolver melhores colaborações, tal qual pode ser observado no trecho que segue:

Acredito que o ecossistema nos trouxe mais empatia com a cadeia de suprimentos. A empresa é grande e poderosa, então ela não se colocava no lugar dos fornecedores. Pelo menos com a área de sustentabilidade, depois que começamos a interagir mais com o ecossistema, aprendemos a nos colocar mais no lugar dos outros.

(Analista de Sustentabilidade, Vareja de moda) 


\section{DISCUSSÃO}

Conforme apresentado anteriormente, este estudo empregou a lente de ecossistemas de inovação para analisar como as inovações ambientalmente sustentáveis ajudam as empresas a desenvolver competências por meio da interação com atores externos. Como uma abordagem de inovação aberta, a visão de ecossistemas de inovação parte da ideia de que inovações são criadas a partir de um "diálogo com múltiplos constituintes” (RITALA; ALMPANOPOULOU, 2017). Portanto, compreender a ligação entre os atores (ou constituintes do diálogo) deste ecossistema é essencial para ter noção dos limites e impactos do ecossistema dentro da empresafocal (MARCON; RIBEIRO, 2021).

Assim, esse estudo propõe que as inovações ambientalmente sustentáveis diferem das inovações regulares e, portanto, criam diferentes ecossistemas de inovação pois precisam considerar os aspectos de sustentabilidade durante o processo de inovação (MARCON et al., 2021). Embora os achados de Marcon et al. (2021) mostrem que os atores no ecossistema em si pouco diferem daqueles esperados em qualquer ecossistema de inovação (como universidades, fornecedores e governo), nossos achados dão conta de que as relações entre os atores do EIAS e a empresa-focal diferem amplamente. Além disso, usando a visão de inovação aberta do EIAS, os resultados mostram que o EIAS ajuda as empresas-focais a desenvolverem competências. Essas competências podem ser técnicas e melhorar a sustentabilidade por meio de materiais, processos ou práticas. Eles também podem ser competências de mercado e inovação para melhorar as habilidades para desenvolver e comercializar a inovação. Além disso, os achados reportados mostram que o EIAS pode melhorar as competências de colaboração das empresas para ajudá-las a explorar melhor a participação em um ecossistema.

A partir das entrevistas, os resultados demonstram que EIAS também agregam competências de sustentabilidade à empresa-focal. Este é um achado relevante pois demonstra que o estabelecimento de parcerias com atores do ecossistema pode não apenas auxiliar as empresas no desenvolvimento de produtos e processos sustentáveis, mas pode também ser o principal responsável-chave para que a inovação verde realmente ocorra (como no caso Simulação em saúde).

\section{CONCLUSÃO}


Esta pesquisa abordou as competências dos ecossistemas de inovação ambientalmente sustentáveis. A contribuição deste artigo para a literatura sobre inovação verde e sobre a teoria dos ecossistemas de inovação diz respeito às competências integradas nas empresas devido à participação em EIAS. Nossos resultados indicam que EIAS pode aprimorar cinco competências principais nos processos de gestão da inovação das empresas, a saber: competências técnicas relativas ao produto, composição de materiais e processos de fabricação; competências de inovação que abordam o nível de maturidade das empresas para desenvolver inovações; competências de mercado que abrangem a capacidade das empresas de atender com sucesso às necessidades dos consumidores e comercializar inovações de acordo com a demanda; competências de sustentabilidade; e, por fim, constatamos que as competências de colaboração são desenvolvidas a partir do EIAS. Ou seja, as empresas aprimoram suas habilidades para colaborar e explorar melhor os resultados do EIAS.

\section{AGRADECIMENTOS}

Os autores agradecem ao CNPq pelo suporte ao estudo realizado na forma de bolsas de pesquisa (Processos Número 140006/2019-2 e 308723/2017-1).

\section{REFERÊNCIAS}

ADNER, R. Match your innovation strategy to your innovation ecosystem. Harvard Business Review, Apr. 2006.

ADNER, R.; KAPOOR, R. Value creation in innovation ecosystems: how the structure of technological interdependence affects firm performance in new technology generations. Strategic Management Journal, v. 31, n. 3, p. 306-333, 2010. DOI:10.1002/smj.821

BEN ARFI, W.; HIKKEROVA, L.; SAHUT, J.M. External knowledge sources, green innovation and performance. Technological Forecasting and Social Change, v.129, p.210 220, 2018. DOI:10.1016/j.techfore.2017.09.017

BOONS, F.; MONTALVO, C.; QUIST, J.; WAGNER, M. Sustainable innovation, business models and economic performance: an overview. Journal of Cleaner Production, v.45, p.18, 2013. DOI: 10.1016/j.jclepro.2012.08.013

CHEN, J.; LINLIN L. Customer participation, and green product innovation in SMEs: the mediating role of opportunity recognition and exploitation. Journal of Business Research, v. 119, p. 151-162, 2019. DOI: https://doi.org/10.1016/j.jbusres.2019.05.033.

MEDEIROS, J.F.; LAGO, N.C.; COLLING, C.; RIBEIRO, J.L.D.; MARCON, A. Proposal of a novel reference system for the green product development process (GPDP). Journal of Cleaner Production, v. 187, p. 984-995, 2018. DOI: 10.1016/j.jclepro.2018.03.237

EDMONDSON, D.L.; KERN, F.; ROGGE, K.S. The co-evolution of policy mixes and sociotechnical systems: towards a conceptual framework of policy mix feedback in sustainability transitions. Research Policy, p. 1-14, 2018. DOI: 10.1016/j.respol.2018.03.010 
EISENHARDT, K.M.; GRAEBNER, M.E. Theory building from cases: opportunities and challenges. Academy of Management Journal, v. 50, p. 25-32, 2007. DOI: $10.2307 / 20159839$

FAGERBERG, J. Mobilizing innovation for sustainability transitions: a comment on transformative innovation policy. Research Policy, v. 47, p. 1568-1576, 2018. DOI: 10.1016/j.respol.2018.08.012

FAGERBERG, J.; MOWERY, D.C.; NELSON, R.R. (Ed.). The Oxford handbook of innovation. Oxônia: Oxford University Press, $2006 . \quad$ DOI: 10.1093/oxfordhb/9780199286805.001.0001

GOMES, L.A.V.; FACIN, A.L.F.; SALERNO, M.S.; IKENAMI, R.K. Unpacking the innovation ecosystem construct: evolution, gaps and trends. Technological Forecasting and Social Change, v. 136, p. 30-48, 2018. DOI: 10.1016/j.techfore.2016.11.009

GRANSTRAND, Ove; HOLGERSSON, Marcus. Innovation ecosystems: A conceptual review and a new definition. Technovation, v. 90, p. 102098, 2020.

JØRGENSEN, U. Mapping and navigating transitions - the multi-level perspective compared with arenas of development. Research Policy, v. 41, p. 996-1010, 2012. DOI: 10.1016/j.respol.2012.03.001

KETOKIVI, M.; TURKULAINEN, V.; SEPPÄLÄ, T.; ROUVINEN, P.; ALI-YRKKÖ, J. Why locate manufacturing in a high-cost country? A case study of 35 production location decisions. Journal of Operations Management, v. 49-51, p. 20-30, 2017. DOI: 10.1016/j.jom.2016.12.005

LEE, C.-W. Strategic alliances influence on small and medium firm performance. Journal of Business Research, v.60, p.731-741, 2007. DOI: 10.1016/j.jbusres.2007.02.018

MARCON, A.; MEDEIROS, J.F.; RIBEIRO, J.L.D. Innovation and environmentally sustainable economy: Identifying the best practices developed by multinationals in Brazil. Journal of Cleaner Production, v. 160, p. 83-97, 2017. DOI: 10.1016/j.jclepro.2017.02.101

MARCON, A.; RIBEIRO, J.L.D.; DANGELICO, R.M.; FRACCASCIA, L. Green innovation ecosystems: an exploratory study of the involved actors. In: INTERNATIONAL JOINT CONFERENCE ON INDUSTRIAL ENGINEERING AND OPERATIONS MANAGEMENT, 26/27., 2021, Rio de Janeiro. Anais [...]. Rio de Janeiro: ABEPRO, 2021.

MARCON, A.; RIBEIRO, J.L.D. How do startups manage external resources in innovation ecosystems? A resource perspective of startups' lifecycle. Technological Forecasting and Social Change, v.171, 2021. DOI: https://doi.org/10.1016/j.techfore.2021.120965

MARKARD, J.; HEKKERT, M.; JACOBSSON, S. The Technological innovation systems framework: response to six criticisms. Environmental Innovation and Societal Transitions, v.16, p.76-86, 2015. DOI: 10.1016/j.eist.2015.07.006

MONTALVO, C.; DIAZ-LOPEZ, F.; BRANDES, F. Potential eco-innovation opportunities in nine sectors of the european economy. Brussels: Consortium Europa Innova Sectorial Innovation Watch, 2011. (Final Report. Task 4 horizontal Report 4)

MOORE, J.F. Predators and prey: a new ecology of competition. Harvard Business Review, v.71, p.75-86, 1993

MOUSAVI, S.; BOSSINK, B.A.G. Firms' capabilities for sustainable innovation: the case of biofuel for aviation. Journal of Cleaner Production, v. 167, p. 1263-1275, 2017. DOI: 
10.1016/j.jclepro.2017.07.146

NAMBISAN, S.; BARON, R.A. Entrepreneurship in innovation ecosystems: entrepreneurs' self-regulatory processes and their implications for new venture success. Entrepreneurship Theory and Practice, v.37, p.1071-1097, 2013

OH, D.S.; PHILLIPS, F.; PARK, S.; LEE, E. Innovation ecosystems: a critical examination. Technovation, v. 54, p. 1-6, 2016. DOI: 10.1016/j.technovation.2016.02.004

RITALA, P.; ALMPANOPOULOU, A. In Defense of "eco" in innovation ecosystem. Technovation, v. 60-61, p.39-42, 2017. DOI: 10.1016/j.technovation.2017.01.004

ROSENTHAL, M. Qualitative research methods : why, when, and how to conduct interviews and focus groups in pharmacy research. Current in Pharmacy Teaching and Learning, v. 8, p. 509-516, 2016.

TSUJIMOTO, M.; KAJIKAWA, Y.; TOMITA, J.; MATSUMOTO, Y. A Review of the ecosystem concept - towards coherent ecosystem design. Technological Forecasting and Social Change, v. 136, p. 49-58, 2018. DOI: 10.1016/j.techfore.2017.06.032

VOSS, C.; TSIKRIKTSIS, N.; FROHLICH, M. Case research in operations management. International Journal of Operations \& Production Management, v. 22, p. 195-219, 2002. DOI: $10.1108 / 01443570210414329$

YAP, X.-S.; TRUFFER, B. Shaping selection environments for industrial catch-up and sustainability transitions: a systemic perspective on endogenizing windows of opportunity. Research Policy, p.1-18, 2018. DOI:10.1016/j.respol.2018.10.002

YAP, X.-S., TRUFFER, B., Shaping selection environments for industrial catch-up and sustainability transitions: A systemic perspective on endogenizing windows of opportunity. Research Policy, 1-18, 2018. doi:10.1016/j.respol.2018.10.002

ZENG, D.; HU, J.; OUYANG, T. Managing innovation paradox in the sustainable innovation ecosystem: a case study of ambidextrous capability in a focal firm. Sustainability, v.9, p.1-15, 2017. DOI:10.3390/su9112091 